\title{
Direct Dark Matter Search with the CRESST II Experiment
}

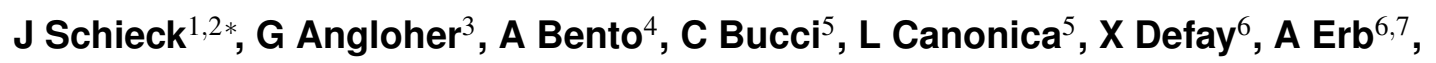
F v Feilitzsch ${ }^{6}$, N Ferreiro lachellini ${ }^{3}$, P Gorla ${ }^{5}$, A Gütlein ${ }^{1,2}$, D Hauff ${ }^{3}$, J Jochum $^{8}$,

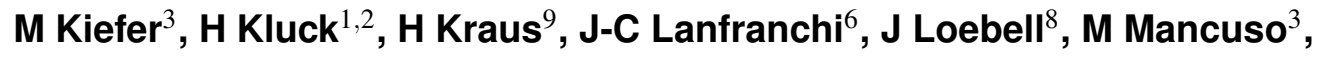
A Münster ${ }^{6}$, C Pagliarone ${ }^{5}$, F Petricca ${ }^{3}$, W Potzel ${ }^{6}$, F Pröbst $^{3}$, R Puig $^{1,2}$, F Reindl $^{3}$, K Schäffner $^{5}$, S Schönert ${ }^{6}$, W Seidel ${ }^{3}$, M Stahlberg ${ }^{1,2}$, L Stodolsky ${ }^{3}$, C Strandhagen ${ }^{8}$, R Strauss ${ }^{3}$, A Tanzke ${ }^{3}$, H H Trinh Thi ${ }^{6}$, C Türkoğlu ${ }^{1,2}$, M Uffinger ${ }^{8}$, A Ulrich ${ }^{6}$, I Usherov ${ }^{8}$, S Wawoczny $^{6}$, M Willers ${ }^{6}$, M Wüstrich $^{3}$ and A Zöller ${ }^{6}$

${ }^{1}$ Institute for High energy Physics, Austrian Academy of Sciences, 1050 Vienna, Austria

${ }^{2}$ Atominstitut, Technische Universität Wien, 1040 Vienna, Austria

${ }^{3}$ Max-Planck-Institut für Physik, D-80805 München, Germany

${ }^{4}$ Departamento de Fisica, Universidade de Coimbra, P3004 516 Coimbra, Portugal

${ }^{5}$ INFN, Laboratori Nazionali del Gran Sasso, I-67010 Assergi, Italy

${ }^{6}$ Physik-Department, Technische Universität München, D-85748 Garching, Germany

${ }^{7}$ Walther-Meißner-Institut für Tieftemperaturforschung, D-85748 Garching, Germany

${ }^{8}$ Eberhard-Karls-Universität Tübingen, D-72076 Tübingen, Germany

${ }^{9}$ Department of Physics, University of Oxford, Oxford OX1 3RH, United Kingdom

The quest for the particle nature of dark matter is one of the big open questions of modern physics. A well motivated candidate for dark matter is the so-called WIMP - a weakly interacting massive particle. Recently several theoretically well-motivated models with dark matter candidates in a mass region below the WIMP mass-scale gained also a lot of interest, theoretically and experimentally. The CRESST II experiment located at the Gran Sasso laboratory in Italy is optimised for the detection of the elastic scattering of these low-mass dark matter particles with ordinary matter. We show the results obtained with an improved detector setup with increased radio purity and enhanced background rejection and the results obtained with a dedicated low-threshold analysis of a single conventional detector module. The limit achieved is the most stringent limit achieved for direct dark matter experiments in the mass region below $1.8 \mathrm{GeV} / c^{2}$. We will discuss the expected performance for new small CRESST-type detectors to be used during the next data taking phase. We conclude with an outlook of the future potential for direct dark matter detection using further improved CRESST $\mathrm{CaWO}_{4}$ cryogenic detectors.

38th International Conference on High Energy Physics

3-10 August 2016

Chicago, USA

${ }^{*}$ Speaker. 


\section{Introduction}

Several observations on different scales clearly point to the existence of dark matter. Up to now dark matter has been observed only via gravitational pull. The existence of a new particle, not being observed by previous and current experiments, offers the best explanation of dark matter. However, the properties of this new particle are yet unknown. Several well motivated theories predict dark matter particle candidates in a large mass range from $\mu \mathrm{eV} / c^{2}$ up to $\mathrm{M}_{\mathrm{GUT}}$. These candidates are expected to interact with ordinary matter with an interaction strength well above the gravitational interaction scale, leading to a possible detection of scattering processes between dark matter and ordinary matter.

Recently dark matter candidates in the mass region of a few $\mathrm{GeV} / c^{2}$ down to the sub-GeV/c region (low-mass dark matter) gained a lot of theoretical interest. This mass region can be still accessed with direct detection experiments, however, the experimental challenges differ from the one experienced by direct detection experiments looking for weakly interacting dark matter (WIMP), located in the mass region of tenth of $\mathrm{GeV} / c^{2}$ to several $\mathrm{TeV} / \mathrm{c}^{2}$. The expected differential scattering rate with respect to the recoil-energy falls exponentially and much more steeply for low-mass dark matter particles compared to particles with larger mass. Direct dark matter experiments looking for WIMPs require a large exposure in order to be sensitive to possible scattering processes. For direct detection experiments, searching for dark matter particles of a few $\mathrm{GeV} / \mathrm{c}^{2}$ or below, the detection energy threshold is crucial, while the total exposure is of lower importance.

The CRESST experiment is a dedicated dark matter direct detection experiment searching for low-mass dark matter particles. In the following we will summarise the latest results obtained by CRESST II and published in $[1,2,3,4]$.

\section{The CRESST II Experiment}

The CRESST II experiment is located at the underground laboratory Laboratori Nazionali del Gran Sasso in Italy. Dark matter particles are detected by looking for elastic scattering processes of dark matter with nuclei of $\mathrm{CaWO}_{4}$ crystals. The experiment is operated at a temperature of about $15 \mathrm{mK}$. The total recoil energy deposited by the scattering process is measured by detecting the generated phonons, using a superconductor attached to the surface of the crystal and operated at its phase transition temperature. A second readout channel measures the scintillating light generated during the scattering process. Scattering of electrons or photons with the crystal produces significantly more light than dark matter scatters (quenching) and the light measurement allows the rejection of background events. For each event the light yield, defined as ratio of the energy measured in the light channel and the phonon channel, is determined. For electron or photon scatters the light yield is normalised to one, scatters of dark matter particles are expected to have a lower light yield. For smaller recoil energies the uncertainty on the light yield increases and background events from electron or photon scattering events can potentially leak into the signal region. The light yield versus phonon energy for data taken with a single detector is shown in Fig. 1. The leakage of background type events into the signal region, indicated by the yellow box, is clearly visible. 


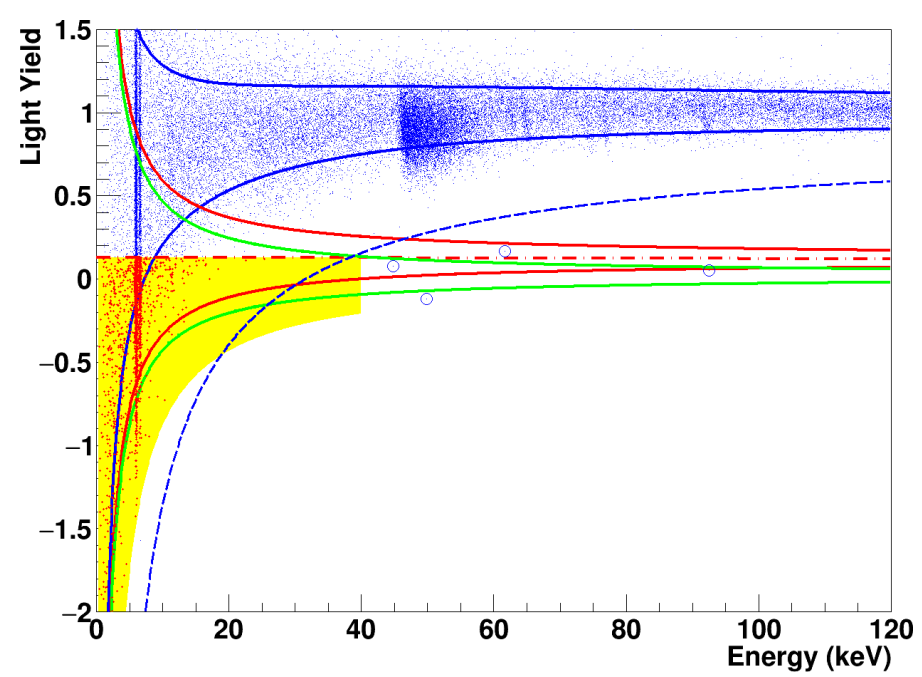

Figure 1: Light yield as a function of the total recoil energy (phonon energy) for the Lise detector [2]. The solid blue line indicates the upper and lower $90 \%$ boundaries for electron or photon scatters, the blue dashed line corresponds to the lower five $\sigma$ boundary. The red (green) lines correspond to the $90 \%$ boundaries for oxygen (tungsten) recoils. The yellow box indicates the signal region. The four events indicated by a circle are statistically incompatible with leakage from the region of electron or gamma scatters and indicate the presence of another source of background events.

The dominant background contribution originates from decays of crystal-intrinsic radioactivity. The overall sensitivity to direct dark matter detection is limited by the number of unidentified background events in the region of interest. Recently the CRESST collaboration started an in-house production of $\mathrm{CaWO}_{4}$ crystals at the Technical University of Munich. The radioactive contaminations are significantly reduced with a background rate as low as 3.5 counts $/(\mathrm{kg} \mathrm{keV} \mathrm{d})$.

\section{Results}

In this article we will mainly discuss results obtained with two different detector modules, called TUM40 and Lise. While the detector module TUM40 [1] is built using a crystal from the in-house production with low intrinsic radioactive noise, the second detector is based on a conventional detection design and a commercial crystal with normal intrinsic background conditions [2]. Data taken during a previous data taking period are used to test the Majorana character of neutrinos [4].

\subsection{Search for low-mass dark matter}

The key for detecting low-mass dark matter particles is the sensitivity for low energetic nuclear recoils. The expected differential dark matter-nucleus interaction rate increases exponentially towards low-mass dark matter particles. The analysis presented here uses data from a single detector module. With the TUM40 detector module a detection threshold of about $600 \mathrm{eV}$ for nuclear recoils is achieved, while for data taken with the Lise detector module the threshold is determined to be about $300 \mathrm{eV}$. The number of selected events in the region of interest is used to set a limit 
on the dark matter-nucleon cross section. For the dark matter density and the dark matter velocity distribution standard astrophysical assumptions are used. A limit is set by assuming all events are originating from dark matter scatters and the maximal possible cross-section is determined by using Yellin's optimal interval method, which is considered to provide a conservative limit.

The result is shown in Fig. 2 and compared to other dark matter searches, using the same astrophysical input. The Lise detector module with a lower energy threshold returns the better limit for lowest mass dark matter particles compared to the result obtained with the TUM40 detector module. For dark matter masses of several $\mathrm{GeV} / \mathrm{c}^{2}$ the limit obtained by experiments based on liquid Xenon are better, since the overall exposure dominates the results. Due to higher background contamination the result of the Lise detector module is worse compared to the limit obtained with the TUM40 detector module. Clearly for dark matter direct detection experiments CRESST II offers the best sensitivity for dark matter candidates below $1.8 \mathrm{GeV} / \mathrm{c}^{2}$.

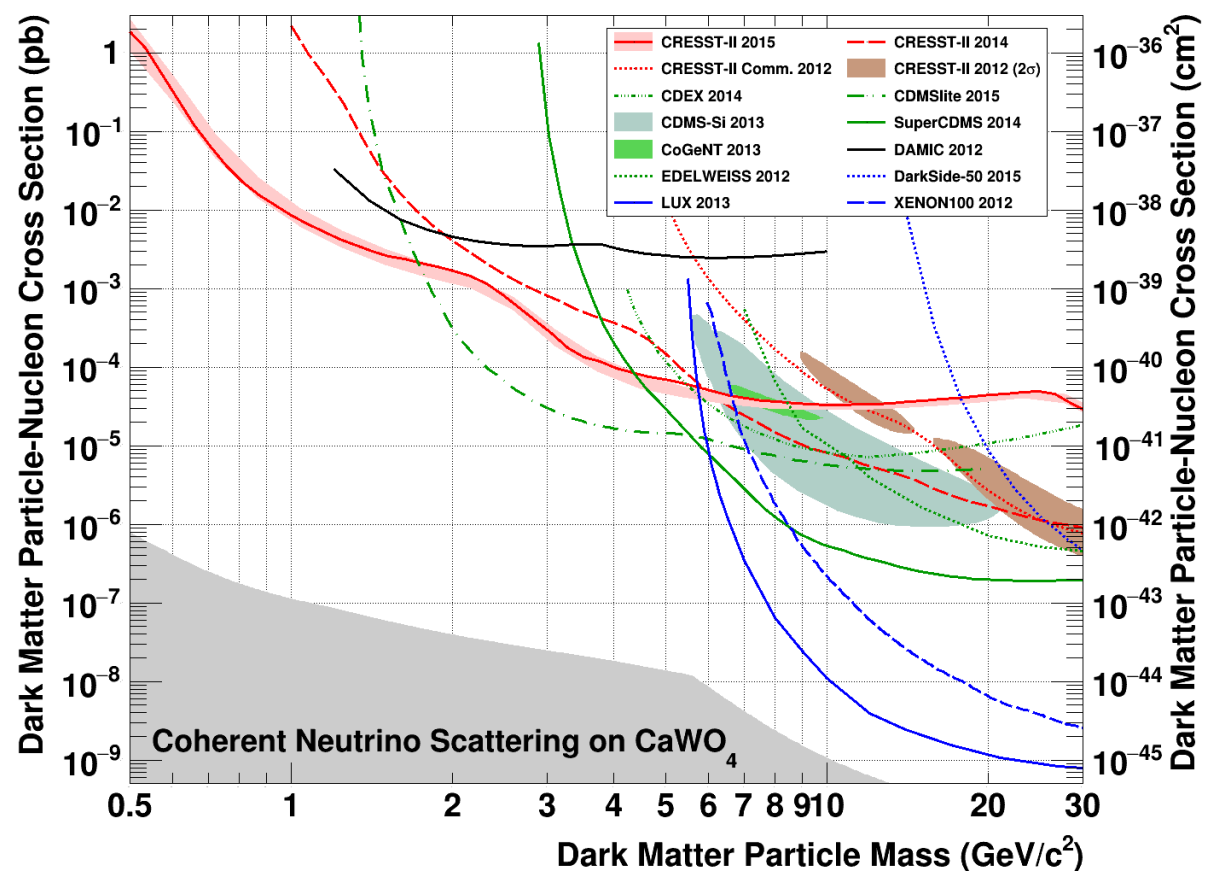

Figure 2: Result of the dark matter searches using CRESST II detector modules [2]. The red dashed line corresponds to the limit obtained with the TUM40 detector module, the solid red line to the limit obtained with the Lise detector module. For further information about the other results shown in the figure the reader is referred to the legend.

\subsection{Limit on the momentum dependent cross-section}

The results summarised in chapter 3.1 assume a momentum independent dark matter-nucleon cross section, leading to an exponential increase of the differential event rate towards low recoil energies. Outstanding problems from comparing helioseismological predictions with solar models can be solved by postulating asymmetric dark matter with a momentum dependent dark matternucleon cross section [5]. The standard cross-section is modified as $\sigma_{\text {dark matter-nucleon }}=\sigma_{0}\left(q / q_{0}\right)^{2}$, 
with $q$ being the momentum transfer, $\sigma_{0}$ the standard momentum-independent cross-section and $q_{0}$ a reference value. This cross-section behaviour leads to a decrease in the expected differential dark matter - nucleon cross section for lower recoil energies. For a momentum transfer of $q_{0}=40 \mathrm{MeV}$, corresponding to a typical recoil energy of $10 \mathrm{keV}$, the discrepancies between the helioseismological predictions and the solar models can be resolved, assuming a dark matter particle with a mass of $3 \mathrm{GeV} / \mathrm{c}^{2}$ and a cross section of $10^{-37} \mathrm{~cm}^{2}$. The data obtained with the Lise detector module can be reinterpreted assuming a momentum dependent cross-section [2]. The limits corresponding to different dependencies on the momentum transfer $q$ are summarised in Fig. 3. The best fit value discussed in [5] can be excluded with the recent CRESST II data set.

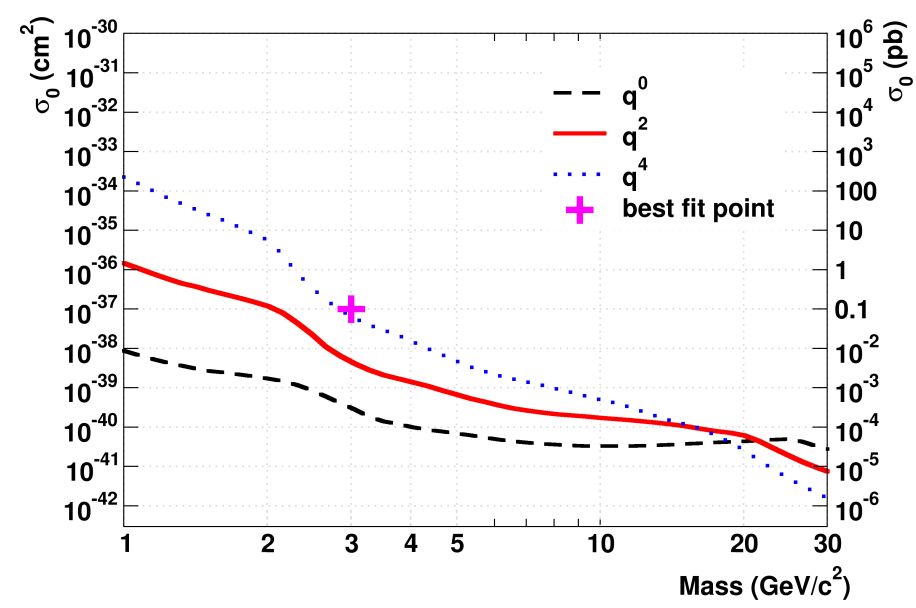

Figure 3: The figure shows the limits for momentum dependent dark matter-nucleon cross sections using data from the Lise detector module [6]. The exponent of the momentum dependent term is summarised in the legend. The best fit value, indicated by the cross, with an asymmetric dark matter model and a $q^{2}$ dependence, as obtained by [5], is excluded.

\subsection{Search for double electron capture processes}

In the Standard Model of particle physics neutrinos are the only possible particles which could act as Majorana-type particles. In this case the particle would be it's own anti-particle. The observation of a simultaneous beta-decay of two nuclei, where the neutrino of one decay is absorbed by the other decay $(0 v 2 \beta)$, would be an unambiguous sign for the Majorana character of neutrinos. Besides this, the Majorana character can also be probed by a neutrino-less double electron capture process $(0 v \mathrm{EC})$, with two electrons simultaneously captured and two protons transformed into neutrons, without emitting any neutrinos. The expected lifetime for such an process is much longer compared to $0 v 2 \beta$ decays and any possible signal is more difficult to observe. However, the current limits for the isotopes ${ }^{40} \mathrm{Ca}$ and ${ }^{180} \mathrm{~W}$ are not very strong and can significantly be improved with the existing CRESST II data set. The double electron capture with two neutrinos released has also not been observed up to now and is part of this analysis as well. In this case the most probable decay is based on the capture of two electrons from the K-shell $(2 v 2 \mathrm{~K})$.

Data from the CRESST II experiment taken between 2009 and 2011 are analysed to search for these processes [4]. While for the results summarised in chapters 3.1 and 3.2 events with 


\begin{tabular}{|c|c|c|}
\hline Isotope & Process & $\mathrm{T}_{1 / 2}[\mathrm{y}]$ \\
\hline${ }^{40} \mathrm{Ca}$ & $0 v \mathrm{EC}$ & $>1.40 \times 10^{22}$ \\
${ }^{40} \mathrm{Ca}$ & $2 v 2 \mathrm{~K}$ & $>9.92 \times 10^{21}$ \\
${ }^{180} \mathrm{~W}$ & $0 v \mathrm{EC}$ & $>9.39 \times 10^{18}$ \\
${ }^{180} \mathrm{~W}$ & $2 v 2 \mathrm{~K}$ & $>3.13 \times 10^{19}$ \\
\hline
\end{tabular}

Table 1: The table summarises the limits for the lifetime $\mathrm{T}_{1 / 2}$, obtained for the two isotopes ${ }^{40} \mathrm{Ca}$ and ${ }^{180} \mathrm{~W}$ and the two different decay scenarios $0 v \mathrm{EC}$ and $2 v 2 \mathrm{~K}$ [4].

a light yield smaller than one are used, here events consistent with an electron or gamma scatter and a light yield of around one are studied. For $0 v \mathrm{EC}$ events the total energy is absorbed in the detector, since no energy is carried away by any neutrino. A potential signal would show up as a peak for electron or gamma events with the expected energy release. No signal is observed and a limit is set for $0 v \mathrm{EC}$ and $2 v 2 \mathrm{~K}$ decays for ${ }^{40} \mathrm{Ca}$ and ${ }^{180} \mathrm{~W}$ isotopes. The limits determined for the different decays and isotopes are summarised in table 1 . For the $2 v 2 \mathrm{~K}$ of ${ }^{180} \mathrm{~W}$ the limit improves by a factor of 30 , compared to previous measurements, the other measurements improve the limit up to a factor of seven.

\section{Outlook}

Recently a new data taking campaign with an improved CRESST II detector module design started. The new design is based on smaller $\mathrm{CaWO}_{4}$ crystals, leading to an even higher phonon density and an increased sensitivity towards smaller nuclear recoil energies. The design goal is to reach an energy threshold of $100 \mathrm{eV}$. Modules are already installed in the cryostat and the detectors reached their operation temperature.

In a second operation phase the sensitivity will be further improved by lowering the intrinsic radioactive background of the crystals by a factor of 100 . The expected sensitivity for the the final operation phase is shown in Fig. 4.

\section{Summary}

Several astrophysical measurements clearly indicate the existence of dark matter. The most likely scenario is a new type of particle. Dedicated experiments search for elastic scattering of these dark matter particles with ordinary matter, CRESST II is one among them. The CRESST II experiment has the best sensitivity to detect low-mass dark matter particles in the masse region between $500 \mathrm{MeV} / \mathrm{c}^{2}$ and $1.8 \mathrm{GeV} / \mathrm{c}^{2}$. The CRESST collaboration recently started an upgrade program to further improve the sensitivity in this mass region by several orders of magnitudes.

\section{References}

[1] G. Angloher et al. [CRESST-II Collaboration], Eur. Phys. J. C 74, no. 12, 3184 (2014) [arXiv:1407.3146 [astro-ph.CO]]. 


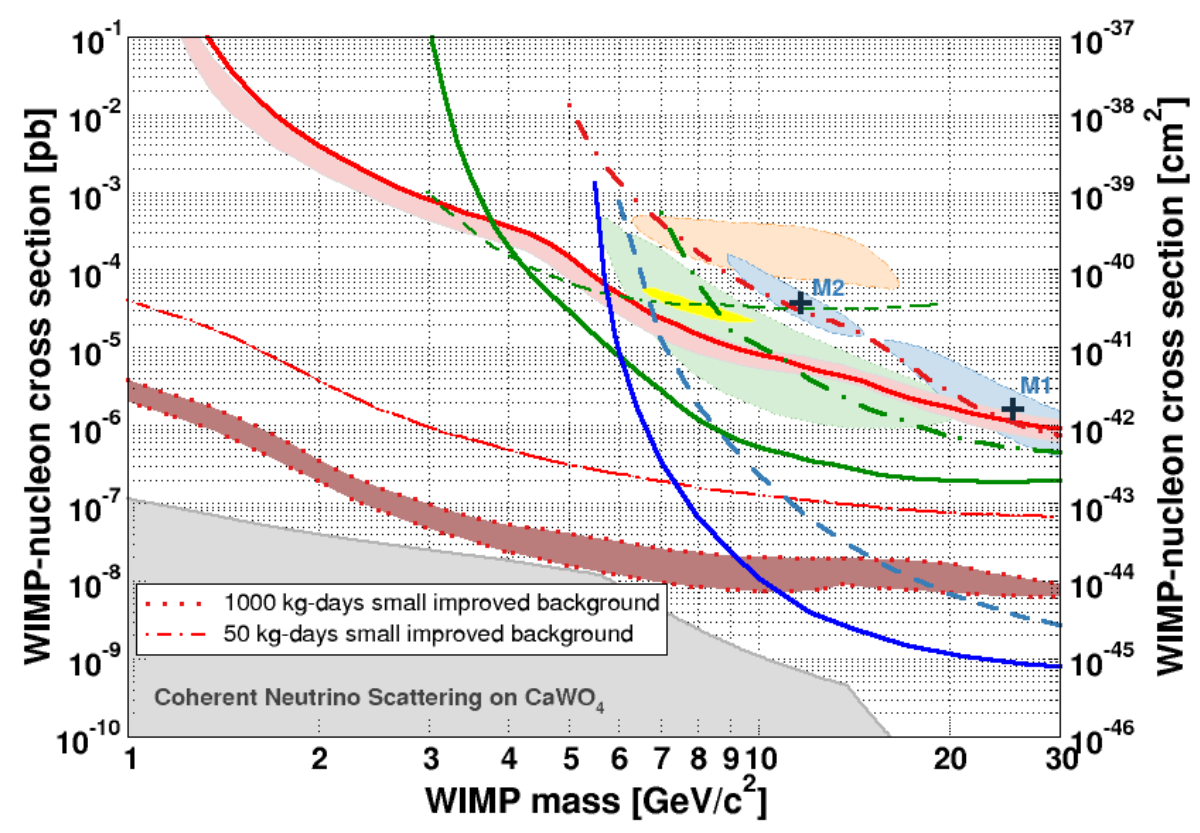

Figure 4: Estimated sensitivity for dark matter detection using the CRESST experiment with an improved detector design with an expected nuclear recoil energy threshold of $100 \mathrm{eV}$ and improved intrinsic radio purity by a factor of hundred [6]. The dashed-dotted (dotted) line corresponds to an exposure of $50 \mathrm{~kg}$-days (1000 kg-days).

[2] G. Angloher et al. [CRESST Collaboration], Eur. Phys. J. C 76, no. 1, 25 (2016) doi:10.1140/epjc/s10052-016-3877-3 [arXiv:1509.01515 [astro-ph.CO]].

[3] G. Angloher et al., Phys. Rev. Lett. 117, no. 2, 021303 (2016) doi:10.1103/PhysRevLett.117.021303 [arXiv:1601.04447 [astro-ph.CO]].

[4] G. Angloher et al., J. Phys. G 43, no. 9, 095202 (2016) doi:10.1088/0954-3899/43/9/095202 [arXiv:1604.08493 [nucl-ex]].

[5] A. C. Vincent, P. Scott and A. Serenelli, Phys. Rev. Lett. 114, no. 8, 081302 (2015) doi:10.1103/PhysRevLett.114.081302 [arXiv:1411.6626 [hep-ph]].

[6] G. Angloher et al. [CRESST Collaboration], arXiv:1503.08065 [astro-ph.IM]. 